\title{
FATORES PREDISPONENTES PARA O INSUCESSO DA CATETERIZAÇÃO INTRA VENOSA PERIFÉRICA EM UNIDADE DE CLÍNICA MÉDICA PEDIÁTRICA
}

\author{
$\underline{\text { Shirlene Cerqueira dos Santos }}{ }^{1}$; Luciano Marques dos Santos ${ }^{2}$; Camila Curcino \\ Santos $^{3}$; André Henrique do Vale Almeida 4 . \\ 1. Bolsista PIBIC/CNPq, Graduanda em Enfermagem pela Universidade Estadual de Feira de Santana, e-mail: \\ shirlene_cerqueira@hotmail.com. \\ 2. Orientador, Departamento de Saúde, Universidade Estadual de Feira de Santana, e-mail: lucmarxenfo@yahoo.com. \\ 3. Bolsista PROBIC/UEFS, Graduanda em Enfermagem pela Universidade Estadual de Feira de Santana, e-mail: \\ camilacurcino@hotmail.com. \\ 4. Docente do curso de Enfermagem, Departamento de Saúde, Universidade Estadual de Feira de Santana, e-mail: \\ almeida_ahv@hotmail.com.
}

PALAVRAS-CHAVE: Enfermagem pediátrica; Punção intravenosa periférica; Criança hospitalizada.

\section{INTRODUÇÃO}

A cateterização intravascular periférica (CIP) constitui uma das intervenções mais frequentemente executadas em pacientes hospitalizados. Para sua consecução, é necessário o desenvolvimento de competência clínica e habilidade técnica dos profissionais de enfermagem, a fim de que sejam preparados para implementar os variados aspectos da terapia intravascular (AVELAR et al., 2010).

A obtenção do acesso vascular periférico na primeira tentativa de punção e a permanência do cateter até o término da indicação da terapia intravenosa devem ser considerados padrão ouro para a sua implementação. Por isso, a manutenção do cateter intravenoso periférico pelo maior tempo possível, livre de complicações e enquanto for indispensável, é um fator importante para minimizar o desconforto da criança e a necessidade de novas punções (AVELAR; PETERLINI; PEDREIRA, 2013).

Entretanto, em alguns momentos, os trabalhadores de enfermagem atuantes em unidades pediátricas podem se deparar com certas condições que podem dificultar a CIP, demandando várias tentativas para a inserção do cateter na rede vascular da criança ou mesmo não conseguindo estabelecer uma via para o início da terapia intravenosa. Esta situação causará desconforto e estresse tanto para a criança quanto para seu familiar acompanhante.

A prevalência do insucesso, conforme dados de pesquisas nacionais e internacionais varia de 4,2\% a 47,8\% (RIKER, et al., 2011; NEGRI et al., 2012; PETERSON et al., 2012; REIGART et al., 2012; AVELAR; PETERLINI; PEDREIRA, 2013). Entretanto, ainda permanecem não esclarecidos os fatores que podem contribuir para a ocorrência deste evento em unidades pediátricas.

Diante disto, fez-se o seguinte questionamento: que fatores podem predispor ao insucesso da CIP em crianças hospitalizadas na unidade de clínica médica?

Este estudo teve como objetivo geral, verificar os fatores predisponentes para o insucesso da CIP realizada em crianças hospitalizadas em unidade de clínica médica pediátrica e como objetivos específicos, caracterizar as crianças hospitalizadas com insucesso na CIP quanto ao perfil sóciodemográfico, o motivo de internação, características da CIP e da terapia intravenosa utilizada e descrever a associação entre características da criança, da terapia intravenosa prévia e da CIP atual com a ocorrência de insucesso na inserção do dispositivo intravascular periférico.

\section{MATERIAIS E MÉTODOS}


Tratou-se de um estudo do tipo transversal, realizado no Hospital Estadual da Criança (HEC) situado na cidade de Feira de Santana, Bahia, especificamente na Clínica Médica. Participaram da pesquisa, 222 crianças e adolescentes que necessitaram de CIP para a implementação da terapia intravenosa como parte de seu tratamento. Os dados foram coletados no período de fevereiro a agosto de 2017 através da observação do procedimento realizado pelo profissional, buscando informações sobre condições da rede venosa, método de punção utilizado e as características da criança, onde foram anotadas em um formulário com as variáveis relacionadas à criança, à terapia intravenosa prévia e CIP e também através da utilização do prontuário de cada criança selecionada.

Os dados foram tabulados em planilhas eletrônicas e analisados através do programa Statistical Package for Social Sciences (SPSS), versão 22.0. Para descrever as variáveis categóricas utilizou-se distribuições de frequências absolutas e relativas e para as variáveis numéricas as médias e o desvio-padrão. As associações entre as variáveis de exposição e desfecho foram avaliadas através de modelos lineares generalizados, calculando-se a razão de prevalência (RP) com intervalo de confiança de 95\% para a não obtenção do acesso intravenoso em relação a uma referência.

O estudo está vinculado à pesquisa intitulada "Segurança do paciente pediátrico e sua família: estudo das tecnologias e eventos adversos relacionados à terapia intravascular periférica". Os responsáveis pelas crianças assinaram o termo de consentimento livre e esclarecido e aquelas com idade superior a sete anos o termo de assentimento, conforme Resolução 466/12 do Conselho Nacional de Saúde. O profissional executor do procedimento, técnicos, auxiliares de enfermagem e enfermeiros, também foi informado antes da observação do procedimento que a pesquisa não implicaria sobre qualquer tipo de avaliação sobre sua prática, nem estaria sujeito a críticas ou sanções administrativas.

\section{RESULTADOS E DISCUSSÃO}

No presente estudo, ao analisar os fatores predisponentes para o insucesso da CIP e comparando-o ao grupo que obteve sucesso, nota-se que a prevalência de insucesso foi de $23,9 \%$, sendo que em pesquisas realizadas anteriormente, a taxa de insucesso variou de 4,2\% a 47,8\%, mas os fatores determinantes para tal não estão bem esclarecidos na literatura (JACOBSON; WINSLOW, 2005; CHINNOCK; THORNTON; HENDEY, 2007; HESS, 2010; RIKER et al., 2011; NEGRI et al., 2012; PETERSON et al., 2012; REIGART et al., 2012; AVELAR; PETERLINI; PEDREIRA, 2013).

O motivo pelo qual esses insucessos aconteceram foi na sua maioria por transfixação da veia, sendo classificado em $26(55,3 \%)$ dos casos. E a maioria desses insucessos aconteceram por 2 tentativas $(34,0 \%)$ e 3 ou mais tentativas $(53,2 \%)$. Com relação às crianças que obtiveram insucesso, (47), 72,3\% eram lactentes (0-24 meses), $61,71 \%$ do sexo masculino, $78,3 \%$ pretos ou pardos, $82,6 \%$ foram classificadas como eutróficas, 93,6\% não tinham histórico de prematuridade, 73,9\% não tinha doença crônica e $76,1 \%$ não estavam com edema no local da cateterização. Destas crianças, $54,3 \%$ foram consideradas agitadas durante a realização da cateterização intravenosa periférica, 70,2\% estavam hospitalizadas por mais de 4 dias hospitalizadas, 64,3\% com diagnóstico de infecção e 82,9\% não tiveram tratamento por tempo prolongado.

No que tange as características da TIV prévia, 76,6\% utilizaram o cateter intravenoso periférico, $80,9 \%$ tinham histórico de dificuldade na inserção deste cateter, $51,1 \%$ não estavam fazendo uso da TIV por tempo prolongado, 83,0\% possuíam histórico de antecedentes de complicações, ou seja, 41,0\% já desenvolveram flebite, 
66,7\% infiltração, 43,6\% extravasamento e 35,1\% obstrução. Com relação à TIV atual, em 83,0\% utilizou-se os membros superiores para realização da CIP, 38,3\% não tinha a rede venosa visível, 40,4\% não era palpável, 39,1\% era tortuosa, $63 \%$ superficial, $19,6 \%$ móvel, $76,6 \%$ foram puncionadas com cateteres de calibre $24 \mathrm{G}$ e $59,6 \%$ utilizaram o método direto.

Foram observadas diferenças estatísticas entre os grupos com e sem insucesso com relação à idade $(\mathrm{x} 2=0,03)$, presença de edema $(\mathrm{x} 2=0,003)$, história de dificuldade na inserção da PIP (x2=0,001) e antecedente de extravasamento (x2=0,044), palpabilidade da rede venosa $(\mathrm{x} 2=0,000)$, trajeto da rede venosa $(\mathrm{x} 2=0,000)$ e $\mathrm{o}$ método de punção $(\mathrm{x} 2=0,000)$. A prevalência do insucesso entre as crianças com idade até seis anos foi 2,0 vezes maior em comparação às com sete anos ou mais e de 2,3 vezes maior quando as crianças/adolescentes foram puncionadas em membros edemaciados. Em crianças que declararam ter dificuldade na inserção da PIP foi 2,7 vezes maior comparadas aquelas que não apresentavam dificuldade. Crianças que já utilizaram CIP anteriormente apresentavam 0,6 vezes menos chances de ter o insucesso, se comparadas a aquelas que usaram outro cateter previamente. A prevalência de insucesso foi de 1,6 vezes maior nas crianças com antecedente de extravasamento. $\mathrm{O}$ insucesso na rede venosa não palpável foi 2,3 vezes maior em comparação com aquelas que a rede venosa era palpável. A prevalência foi 2,5 vezes maior onde a rede venosa era considerada tortuosa e de 0,3 vezes maior quando a PIP era realizada através do método direto.

As instituições devem considerar as características da população pediátrica em cada unidade de internamento, assim como o método se inserção da PIP, e os protocolos de normas e rotinas da unidade como aquelas normas que regem as tentativas de inserção e o tempo de utilização de um dispositivo (PETERSON et al., 2012), pois a necessidade de múltiplas tentativas de colocar um acesso intravenoso pode atrasar a terapia, aumentar a dor e ansiedade de crianças submetidas ao procedimento, bem como alterar a confianças dos pais ou responsáveis pela criança na qualidade do atendimento (PAIVA, 2015).

Sendo assim, a capacidade que um profissional tem de identificar crianças difíceis de inserção da PIP pode permitir à equipe a estimar ao pai/responsável as tentativas necessárias para a PIP e, possivelmente, quantas tentativas provavelmente serão necessárias para o sucesso (YEN; RIEGERT; GORELICK, 2008).

\section{CONCLUSÃO}

Este estudo possibilitou verificar alguns fatores predisponentes para o insucesso na PIP em crianças hospitalizadas no Hospital Estadual da Criança em Feira de Santana na Bahia. $\mathrm{Na}$ amostra investigada observaram-se diferenças estatisticamente significantes para as variáveis idade, presença de edema, história de dificuldade na inserção do CIP, antecedente de extravasamento, palpabilidade e trajeto da rede venosa e método de punção. Crianças menores de 6 anos, com membros edemaciados, que estavam em uso prolongado de terapia intravenosa, com antecedentes de complicação intravenosa do tipo extravasamento, que tinham veias não palpáveis e tortuosas, através do método direto, tiveram um maior risco de obter o insucesso na inserção da PIP.

A identificação precoce dos pacientes que apresentam dificuldade para inserção de cateter intravenoso periférico ao início da hospitalização e o aperfeiçoamento constante das técnicas de inserção do cateter, facilitam a PIP e aumentam as taxas de sucesso nas primeiras tentativas, contribuindo positivamente para que o hospital reduza gastos desnecessários a cada tentativa de punção, evitando também o estresse da criança e de sua família, provocado pela repetição do procedimento. 
A limitação do estudo foi a incipiente produção científica sobre os fatores predisponentes para o insucesso em crianças. O estudo tem relevância profissional, teórica, e social, pois, os resultados poderão fornecer fundamentos científicos para a prática clínica, não somente para a equipe de enfermagem, como de todos os profissionais de saúde envolvidos no cuidado de crianças com necessidade de PIP, além de promover a reflexão a respeito das técnicas de venopunção atualmente desenvolvidas por estes, podendo implicar em uma transformação das práticas de saúde, visando o conforto e bem estar da criança hospitalizada.

\section{REFERÊNCIAS}

AVELAR, A.F.M et al. Capacitação de enfermeiros para uso da ultrassonografia na punção intravascular periférica. Acta Paul Enferm. v.23, n.3, p.433-6, 2010.

AVELAR, A. F. M.; PETERLINI, M. A. S.; PEDREIRA, M. L. G. Assertividade e tempo de permanência de cateteres intravenosos periféricos com inserção guiada por ultrassonografia em crianças e adolescentes. Rev. esc. enferm. USP. v.47, n.3, p.539546, 2013.

CHINNOCK, B.; THORNTON, S.; HENDEY, G.W. Predictors of success in nurseperformed ultrasound-guided cannulation. The Journal of Emergency Medicine. v. 33, n. 4, p. 401-405, 2007.

JACOBSON, A. F.; WINSLOW, E. H. Variables influencing intravenous catheter insertion difficulty and failure: An analysis of 339 intravenous catheter insertions. Heart \& Lung. v.34, n.5 p.345-359, 2005.

HESS, H.A. A biomedical device to improve pediatric vascular access success. Pediatr Nurs. v.36, p.259-263, 2010.

NEGRI, D.C et al. Fatores predisponentes para insucesso da punção intravenosa periférica em crianças. Rev. Latino-Am. Enfermagem. v.20, n.6, 2012.

PAIVA, L.B.F de. Fatores predisponentes ao insucesso na inserção de dispositivo intravascular periférico em crianças com condições cirúrgicas. Trabalho de Conclusão de Curso (Bacharelado em Enfermagem) - Colegiado de Enfermagem, Universidade Estadual de Feira de Santana, Feira de Santana, 2015.

PETERSON, K.A. et al. Does the use of an assistive device by nurses impact peripheral intravenous catheter insertion success in children? Journal of Pediatric Nursing. v.27, p.134-143, 2012.

REIGART, J. R. et al. Peripheral Intravenous Access in Pediatric Inpatients. Clin. Pediatr. v. 51, n.5, p.468-472, 2012.

RIKER, M. W. et al. Validation and refinement of the Difficult Intravenous Access Score: a clinical prediction rule for identifying children with difficult intravenous access. Acad. Emerg. Med. v.18, n.11, p.1129-1134, 2011.

YEN, K.; RIEGERT, A.; GORELICK, M.H. Derivation of the DIVA Score: A clinical prediction rule for the identification of children with difficult intravenous Access.

Pediatric Emergency Care. v.24 n.3 p.143-147, 2008. 